\title{
Calculation the optical energy band gap of LR115 SSNTD irradiated by $\alpha$ particle
}

\author{
Hassan M.Jaber Al-Tai'i \\ Department of Physics, College of Science, AL-Muthana University \\ E-mail: domez973@yahoo.com
}

\begin{abstract}
The effect of $\alpha$-particle irradiation on the optical absorption in nuclear track detectors (LR115) has been studied. These detectors have been irradiated with different doses. The optical absorption has been measured using the ultraviolet-visible (UV-1100) spectroscopy, that irradiation results in shifting the peaks of the optical absorption. The values of Urbach energy have been calculated from the position of steady-state optical band gap energy, for a standard sample which was unirradiated with indirect influence, has been found $1.9 \mathrm{eV}$ whereas its value after irradiation $1.98 \mathrm{eV}$. In case of the direct influence, it is found to be, respectively, before irradiation $1.98 \mathrm{eV}$ and after irradiation $2.05 \mathrm{eV}$. From these results, we can reveal that the values of energy gaps in direct-coincidence before and after irradiation greater than those for indirect one. The number of carbon atoms has been determined in each case for the optical energy gaps.
\end{abstract}

Key words

LR115, Optical energy gap, $\alpha$ particle.

Article info.

Received: Sep. 2012

Accepted: Jun. 2013

Published: Dec. 2013

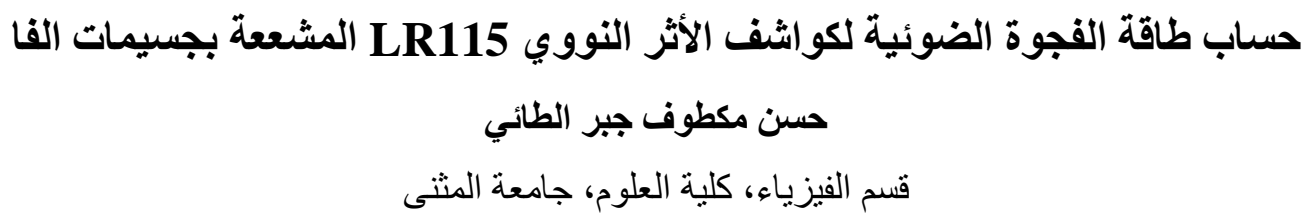

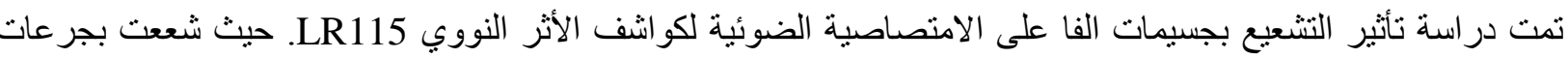

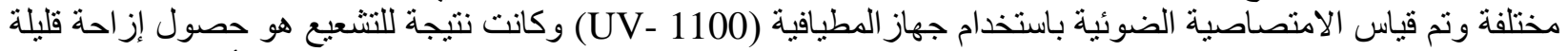

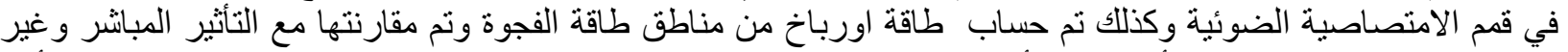
المباثر للعينة القياسية، ووجد أن قيمة التأثير غير المباشر قبل التشعيع 1.9eV وبعد التشعيع 1.98eV وفي حالة التئ التأثير

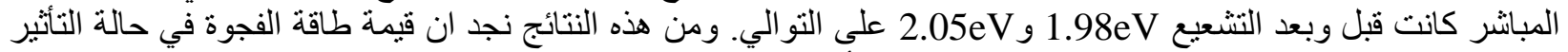

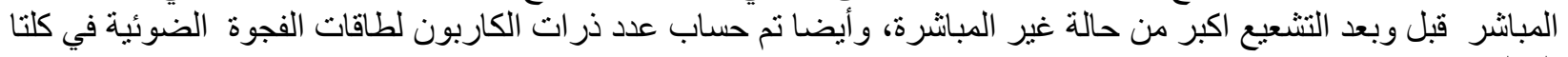

\section{Introduction}

In resent year the solid-state nuclear track detectors (SSNTDs) have wide applications such as for radon and progeny measurements [1]. The optical properties such as grey level, major and minor axes are useful in automatic track measurements [2]. The track effect exists in many materials and it was best manifested in materials with long molecules[3]. The latter being the best solid state nuclear track detectors [4]. Some amorphous materials like glasses, and some natural minerals like mica, apatite or olivine, were demonstrating the track effect. Dielectric materials are the commonest materials were showing the track effect. Many materials were examined and were 
used for commercial manufacturing of detectors [5]. One of the most frequently used detectors is the polyallyldiglycol carbonate (PADC) (commercially available as the CR-39 detector) discovered by Cartwright et al. (1978)[3]. Another frequently used cellulose nitrate as a detector which has the basic material employed for the commercially available LR-115 detector [6]. Other kinds of detectors were commonly in used which include the polycarbonate on which the commercially available Makrofol detector is based [6]. During the last a few decades, the polymers were proven potential in all fields [8]. Different studies on the effect of irradiation on polymers show a variety of modifications in physical and chemical properties. The polymeric materials were depending on the irradiation influence and on radiation energy used. Interaction of the radiation or highly energetic charged ion strikes a polymer target; it loses most of its energy in exciting or ionizing the atoms along its trajectory[7]. Target ionization causes bond cleavage (scission), crosslinking, degradation, emission of atoms and molecules [7]. Ion beam bombardment is a suitable tool to modify the physicochemical properties of polymers (CR39), known polymer, is used in diverse fields such as neutron dosimetry, gamma and cosmic ray detection, heavy ion and nuclear physics [7]. The LR115 type film is sensitive for $\alpha$ particles such that when $\alpha$-particle hits the film it causes localized damage to the molecular structure of the cellulose nitrate layer. This damage can be visibly observed when the exposed film is etched in a bath of diluted sodium hydroxide solution amongst other specific conditions. After this processing small holes are visible under the microscope which is able to be quantitated. The holes exhibit diameters from $1 \mu \mathrm{m}$ to 15 $\mu \mathrm{m}$ and show where the film was hit by $\alpha$ particles. A wide range of colors can be achieved by dyeing the surface or the bulk of the material. The use of ion beams to modify polymer properties opened a wide area of research and utilization in various fields like industry, agriculture, ecology, sensorics, microelectronics and nanotechnology. The damage processes triggered by ions can be different from those induced by classical low ionizing particles as electrons or gamma rays. This is due to the very high electronic stopping power of these ions. A gradual increase in absorbance, generation of chromophoric groups, loss of crystalline, formation of carbonized inclusions along with a gradual phase transition, as well as creation of threedimensional network and triple bonds have been found in polymers due to ion bombardment. In most cases the amount of energy deposited in the host polymer is enough for extensive breaking of the chemical bonds within the track, which makes that strong modification of the bombarded polymer material, is possible. The energy released by the ion during slowing down is deposited in the target by means of two basically different mechanisms, electronic excitation and nuclear collision, which induce quite different processes of polymer matrix rearrangement [10-12].

\section{Experimental Procedure}

LR115 $\left(\mathrm{C}_{12} \mathrm{H}_{17} \mathrm{O}_{16} \mathrm{~N}_{3}\right)$ samples with thickness of $4.5 \mu \mathrm{m}$ were irradiated using different alpha particles doses from ${ }^{210} \mathrm{Po}$ point source in (Al-Muthanna University/ Science Faculty/ Physics Department). The samples $\left(2 \times 1 \mathrm{~cm}^{2}\right)$ were irradiated for various times and the total alpha doses obtained were listed in Table 1 . The nature of optical modifications of pristine and alpha irradiated LR115 polymer samples were subjected to spectral studies in the UV-Visible region. These studies were carried out by using specetro-photometer 
(UV1100) in the wavelength range of 500$840 \mathrm{~nm}$.

\section{Results and Discussion}

The optical absorption spectra of pristine and alpha irradiated LR-115 samples were recorded using UV spectrophotometer in the wavelength range of 500$840 \mathrm{~nm}$ at room temperature. Fig. 1 Show a shift of absorption edge to words longer wavelength with increasing in alpha absorbed dose which can be readily observed. The absorption peak with increasing dose is seen to change into abroad one; the behavior is generally interpreted as caused by the formation of extended systems of conjugate bonds and possible formation of carbon cluster defects. The absorption bands in the investigated range of wavelength area associated to the $\left(\pi-\pi^{*}\right)$ electronic transition. This type of transition occurs in the unsaturated center on the molecules. Which could be containing double or triple bonds and also in aromatics system. The excitation of $(\pi)$ electron requires smaller energy and hence transition of this type occurs at longer wavelengths.

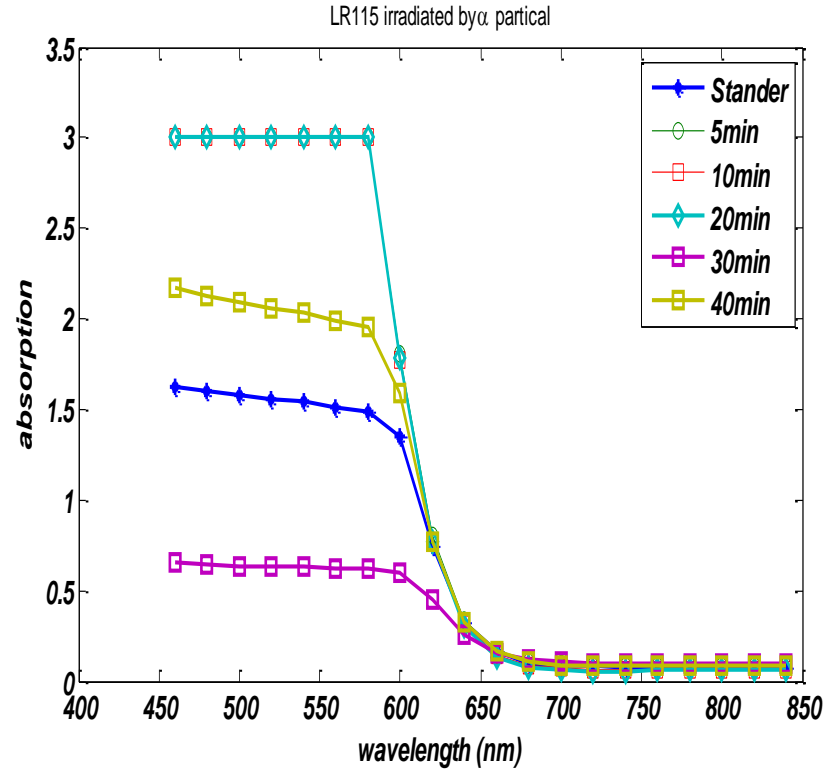

Fig. 1:The visible spectrum of the pristine and alpha-irradiated LR-115.

The optical absorption coefficient $(\alpha)$ was calculated from the absorbance (A) after correction for reflection losses; $(\alpha)$ may be obtained using the following equation [1]:

$\alpha(v)=2.303 A / l$

Where $l$ is the sample thickness in $\mathrm{cm}, \mathrm{A}$ is defined by $\mathrm{A}=\log$ (I/Io) where Io and I are the intensity of incident and transmitted beams, respectively.

Table 1: Variation of band gap energy and Urbach's energy in the pristine and alpha irradiated LR115 with number of carbon atoms $(N)$ per conjugated length

\begin{tabular}{|c|c|c|c|c|c|c|}
\hline \multirow{2}{*}{ Time $(\mathrm{min})$} & \multirow{2}{*}{$\begin{array}{c}\alpha \text { Dose } \\
(\mathrm{mGy})\end{array}$} & \multicolumn{2}{|c|}{ Band gap Energy $(\mathrm{eV})$} & \multirow{2}{*}{$\begin{array}{c}\text { Urbach } \\
\text { energy }\end{array}$} & \multicolumn{2}{|c|}{ No.of carbon atome $(\mathrm{N})$} \\
& & & Indirect & & Direct & Indirect \\
\hline 0 & virgin & 1.98 & 1.9 & 0.06 & 9 & 10 \\
5 & 0.9 & 2.03 & 1.95 & 0.076 & 9 & 9 \\
10 & 1.8 & 2.05 & 1.96 & 0.052 & 9 & 9 \\
20 & 3.6 & 2.05 & 1.96 & 0.059 & 9 & 9 \\
30 & 5.4 & 1.93 & 1.89 & 0.067 & 9 & 10 \\
40 & 7.2 & 1.99 & 1.91 & 0.059 & 9 & 10 \\
\hline
\end{tabular}



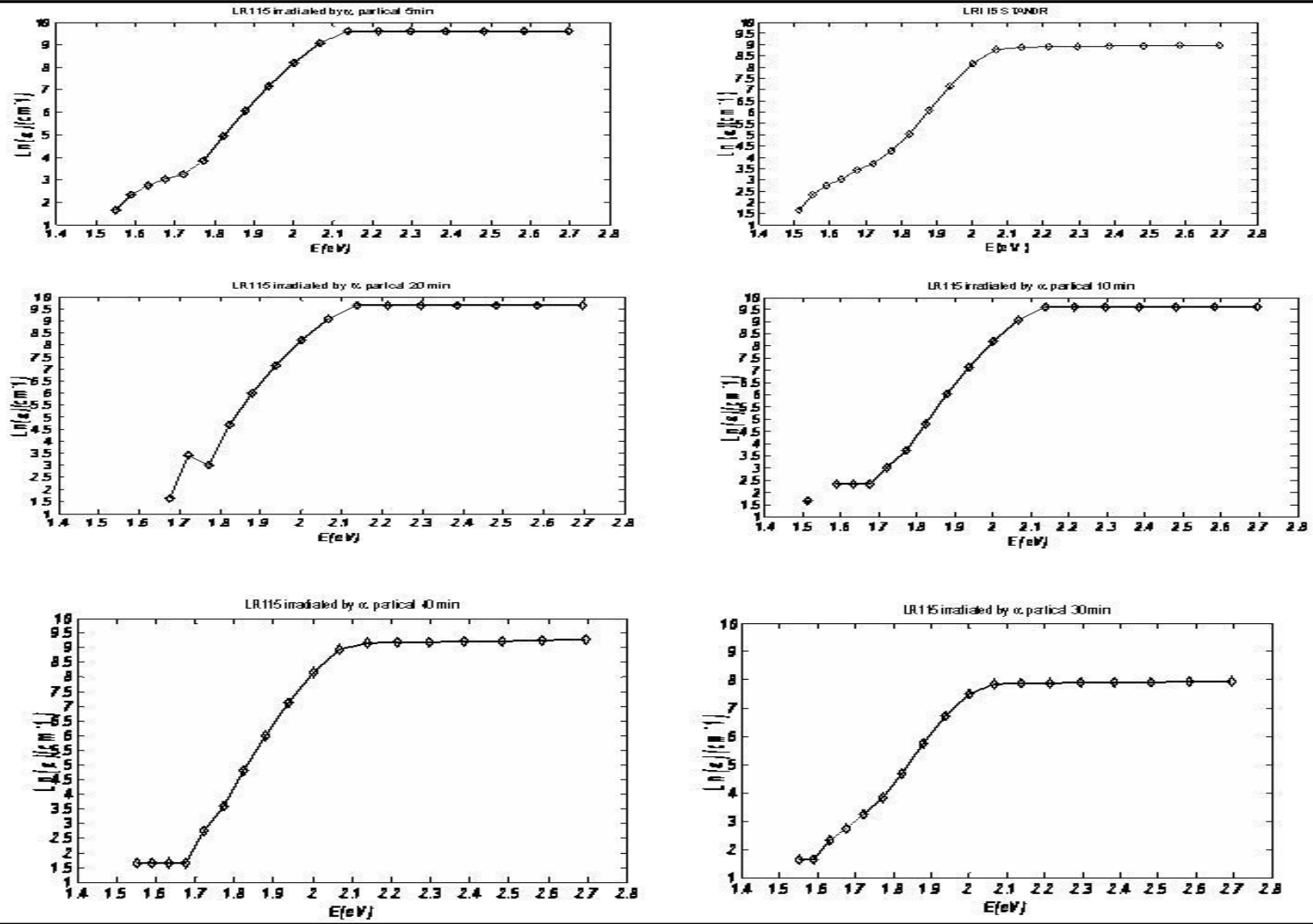

Fig. 2:The dependence of natural logarithm of $\alpha$ on photon energy for pristine and alpha irradiated LR-115 polymer.

The logarithm of the absorption coefficient $\alpha(v)$ was plotted as a function of the photon energy $(h v)$ for LR-115 irradiated with different doses of alpha as in Fig.2. The values of the Urbach's energy (Eu) were calculated by taking the reciprocal of the slopes of the linear portion in the lower photon energy region of these curves as listed in Table1. The decrease in the Urbach's energy in case of LR-115 is due to the decrease in the crystalline nature of the polymer. The Urbach's energy vacillation decreases with increasing alpha doses. This vacillation decrease indicates the irregularization of the band gap energy level; furthermore this is due to amorphous nature of LR115 polymer. The decrease in band gap energy attributes to the decrease in the resistivity of LR-115. This means that there is a change in the structural characteristics of LR-115 as a result of alpha irradiation. The value of energy gap in the case of indirect transitions, in most cases are lower than the energy gaps of direct transition, due the presence of delocalized states between valance and conduction band $[8,10]$. It may accounts for the scission of the polymer chain and formation of free radicals[8]. It is decreasing trend of energy gap with increasing of alpha dose. This is due to the carbon enriched domains created in polymer during the irradiation. In the wavelength rang 500-840 $\mathrm{nm}$ studies, the absorptions are associated with $\pi-\pi^{*}$ electron transition. The excitation of $\pi$ electron requires smaller energy and hence, transition of this type occurs at longer wavelengths. The usual method for determining the value of $E_{g}$ involves 
plotting $(\alpha h v)^{1 / n}$ against $(h v)$. indirect transition in many amorphous materials fits the case for $n=2$; for a direct reasonable fit with $n=1 / 2$ is achieved, In this study, the most satisfactory results were obtained by $\operatorname{plotting}(\alpha h v)^{1 / 2}$ and $(\alpha h v)^{2}$ as a function of photon energy $(h v)$ respectively, taking into account the linear portion of the fundamental absorption edge of the UV- spectrum band gap in polymer, i.e: indirect and direct, have been determined for pristine and alpha irradiation LR-115 polymer as shown in Fig.3 and 4. The values of the indirect and direct band $\operatorname{gap}\left(E_{g}\right)$ were obtained for pristine(virgin) and alpha irradiated sample, respectively.
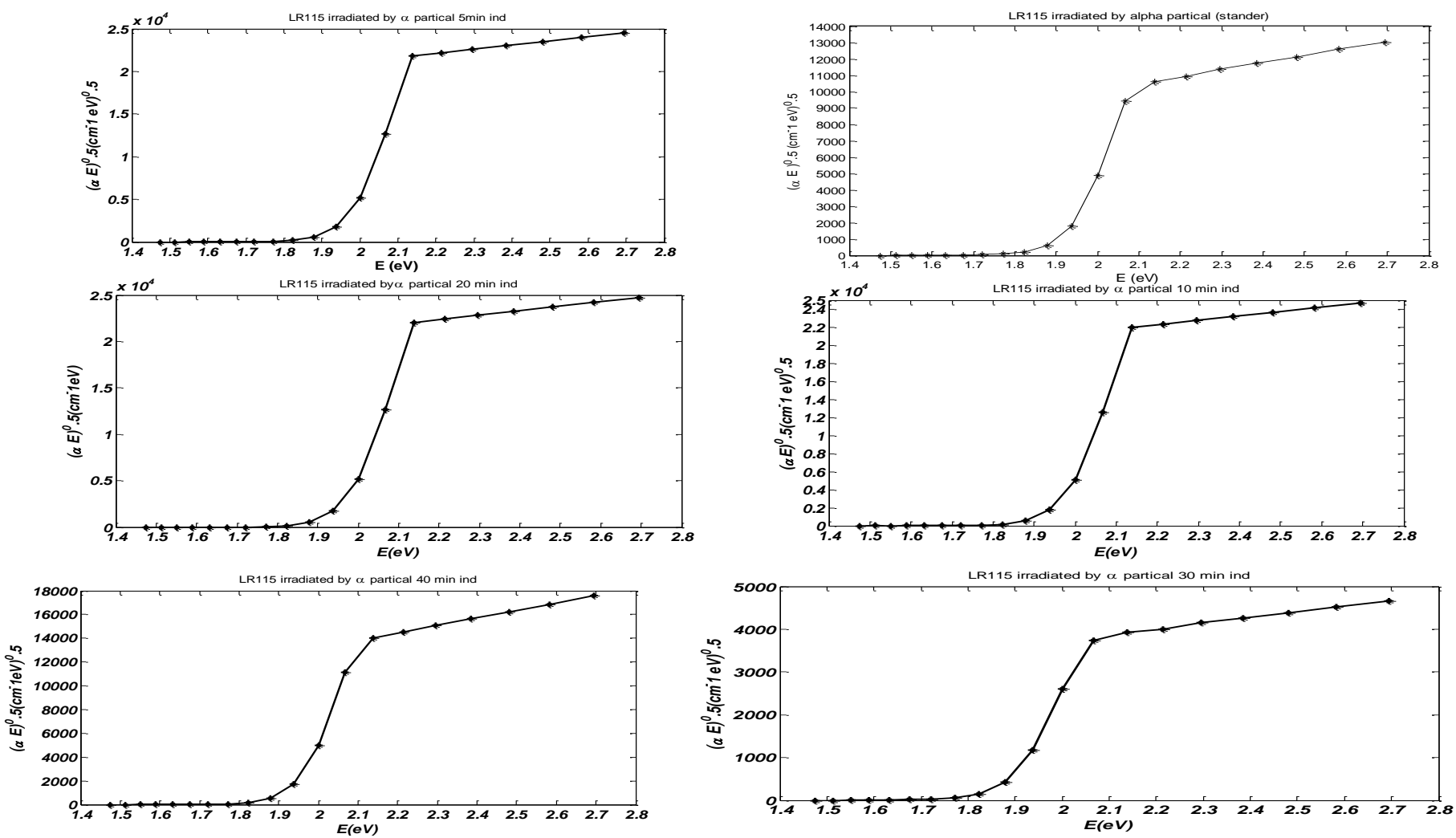

Fig.3: The dependence of (ahv) ${ }^{1 / 2}$ on photon energy (hv) for pristine and alpha irradiated LR-115

Polymer.

Furthermore, the values of indirect band gap have been found to be lower than that the corresponding values for the direct band gap as shown in Fig.5. The simultaneous existence of indirect as well as direct band gap in LR-115 polymer not has been reported; Pristine LR-115 is transparent and has very little absorption, upon irradiation [8]. For a linear structure the number of carbon atoms per conjugation length $N$ is given by[11]:

$$
N=2 \beta \pi / E_{g}
$$

Here $2 \beta$ gives the band structure energy of a pair of adjacent $\pi$ sites. The value of $\beta$ is found to be $2.9 \mathrm{eV}$ [11] as it is associated with $\left(\pi-\pi^{*}\right)$ optical transitions in $(-\mathrm{C}=\mathrm{C}-)$ structure. As the shift of the absorption edge can be attributed to an increase of the conjugation length without formation of new lengthy linear conjugated structures, Eq. 2 is applied in this study. 

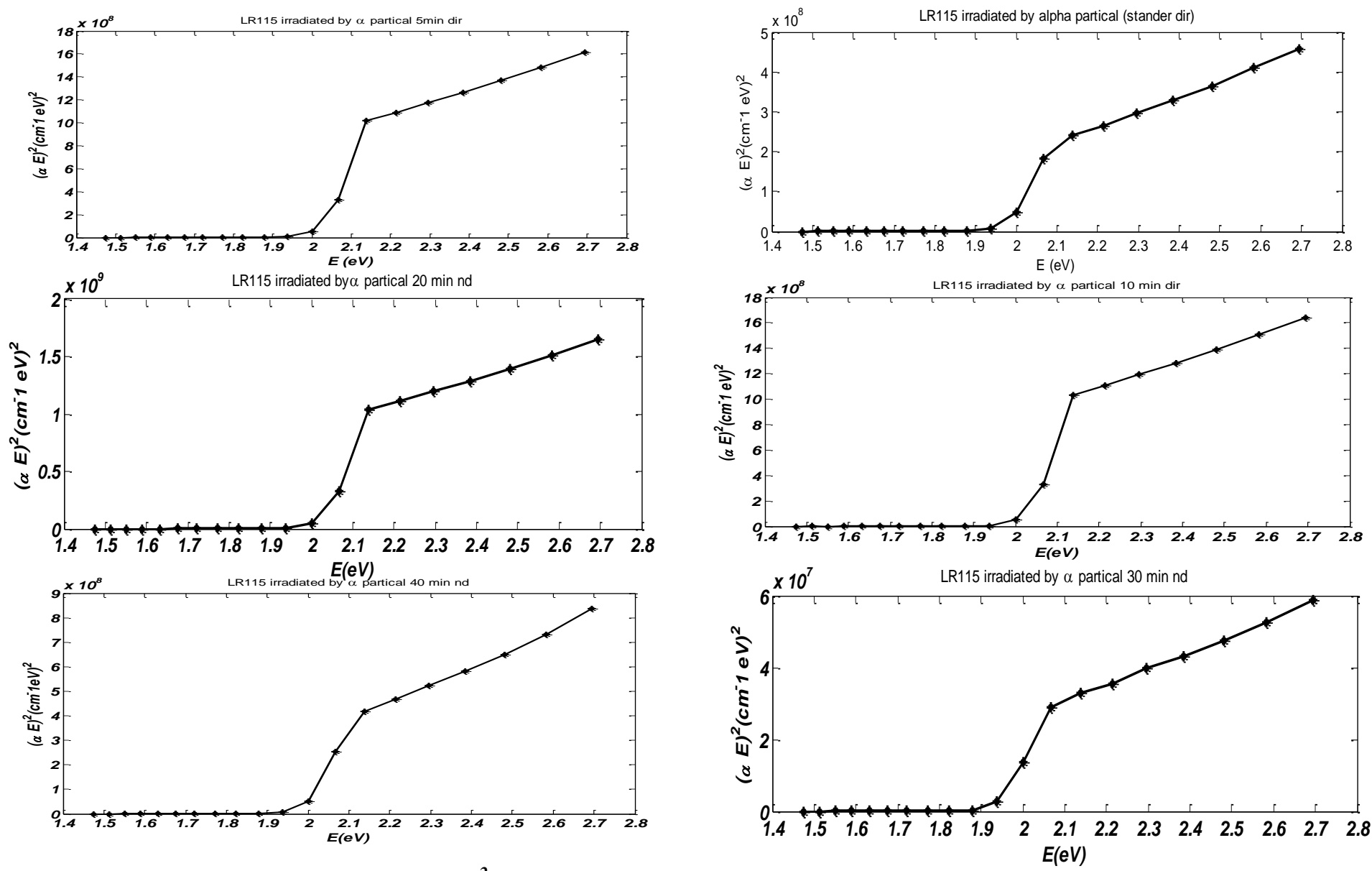

Fig.4: The dependence of $\left((\alpha h v)^{2}\right.$ on photon energy (hv) for pristine and alpha irradiated LR-115

polymer.

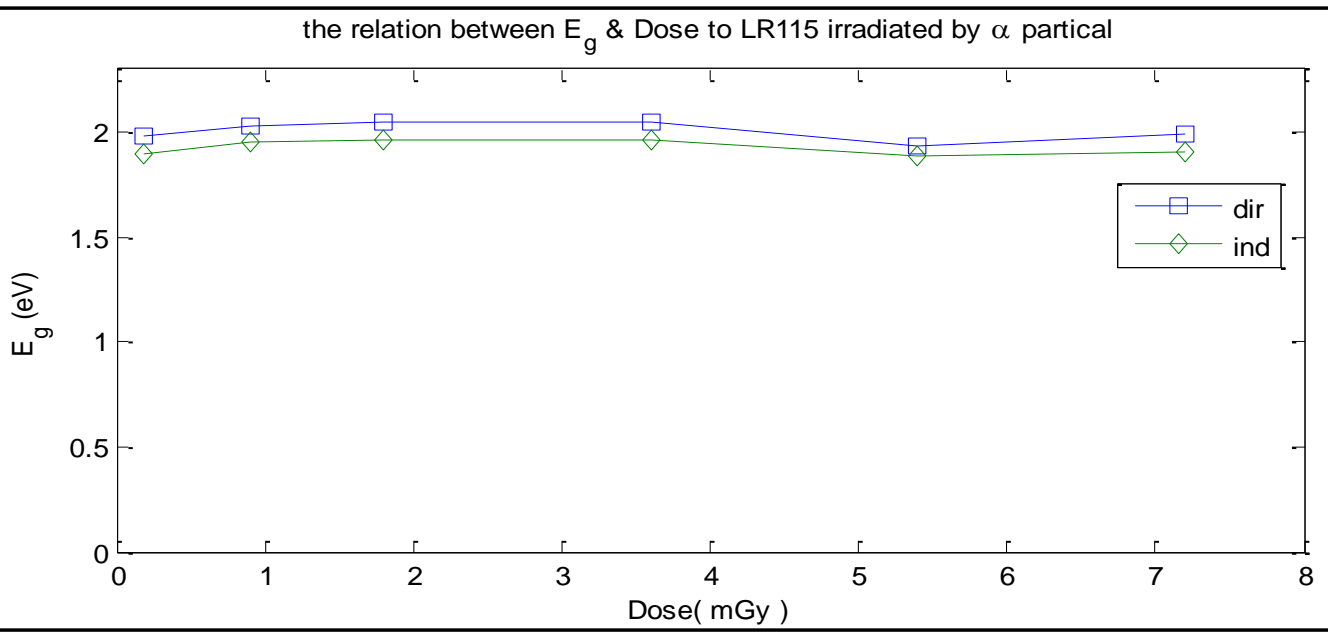

Fig.5: Plots for transition energy (eV) as a function of alpha absorbed dose (mGy).

\section{Conclusions}

A significant change was observed in optical response of the LR-115 polymer after the experimental irradiation of pristine and alpha irradiation LR-115. The values of the optical band gap Eg and Urbach's 
energy $E_{u}$ were determined from the optical absorption spectrum. It is concluded that the values of the indirect band gap are lower than that the corresponding values of the direct band gap in pristine and alpha irradiated LR-115 polymers. Also the optical band gap (Eg) decreases with the increasing of alpha influences which is due to the photon- degradation of LR-115 and the formation of defects and cluster in the material, While the cluster size (number of carbon atoms $(\mathrm{N})$ ) increase with increasing doses for direct influence but constant for indirect influence Finally we found that the energy gap became small greater than in high doses.

\section{References}

[1] Shafi-Ur-Rehman, Ph.D.Thesis, PIEAS, Nilore, Pakistan (2005).

[2] Y. L. Law, D. Nikezic, K.N. Yu, Radiation Measurements, 43 (2008) S128S131.

[3] D. Nikezic, and K.N. Yu, Methods and Applications ISBN: 978-1-60876-826. Nova Science Publishers, Inc, (2009).

[4] K.N. Yu, M. F. Ng, J.P.Y, C.W. Y. Yip and D. Nickez, Radiation Protection Dosimetry, 111,1 (2004) 93-96.
[5] Fleischer, RL: Tracks to innovation, nuclear tracks in science and technology, New York, Springer 1998.

[6] P.C. Kalsi, A. Ramaswami, and V. K. Manchanda, Radiochemistry Division.

[7] M. F. Zaki, Y.H.El-Sharer, Pramana Journal of Physics, 69, 4 (2007) 567-574.

[8] Kumar,Vijayar, R.G.Sonkawade, B A. S.Dhaliwal and Rohtmerea, Asian Journal Of Chemistry, 21, 10 (2009) S279-283.

[9] Kumar, Vijayar, R. G. Somkawade and A.S.Dhaliwal, Indian Journal of Pure and Applied Physics, 48 (2010) 466-469.

[10]Basma A. El-Badry, M. F. Zaki, A. M. Abdul-Kader, Tarek M. Hegazy, A. Ahmed Morsy, Elsever, Vacuum, 83 (2009) 11381142.

[11] M. F. Zaki, Brazil Journal of Physics, 38, 4 (2008) 558-562.

[12] A. S. Roussetski, Lebedev Physical Institute, Russian Academy of Sciences, Condensed Matter Nuclear Science, Proceedings of the 11th International Conference on Cold Fusion. Held 31 October-5 November 2004 in Marseilles, France, 2006. ISBN \#9789812774354, 274280. 